\title{
A study to assess the feasibility of undertaking a randomized controlled trial of adherence with eye drops in glaucoma patients
}

This article was published in the following Dove Press journal:

Patient Preference and Adherence

4 October 2013

Number of times this article has been viewed

\author{
Cliff Richardson' \\ Lisa Brunton' \\ Nicola Olleveant' \\ David B Henson' \\ Mark Pilling' \\ Jane Mottershead ${ }^{2}$ \\ Cecilia H Fenerty ${ }^{2}$ \\ Anne Fiona Spencer ${ }^{2}$ \\ Heather Waterman' \\ 'School of Nursing, Midwifery and \\ Social Work, University of Manchester, \\ ${ }^{2}$ Royal Manchester Eye Hospital, \\ Central Manchester Foundation Trust, \\ Manchester, United Kingdom
}

Background: Adherence with therapy could influence the progression of glaucoma and ultimately affect the onset of visual impairment in some individuals. This feasibility study evaluated the measures to be used for a future randomized controlled trial assessing the effects of group-based education on adherence with eye drops.

Methods: People diagnosed with glaucoma within the previous 12 months attending a regional ophthalmology clinic in the North West of England were recruited. A two-session education program delivered one week apart had been devised as part of a previous project. A combined adult learning and health needs approach to education was taken. Outcomes measured were knowledge of glaucoma, self-report of adherence, illness perception, beliefs about medicines, patient enablement, and general health (Short Form-12). Adherence was also measured objectively using a Medical Events Monitoring System device.

Results: Twenty-six participants consented to undertake the educational program and 19 produced analyzable data. Knowledge of glaucoma, illness perception, beliefs about medicine, and patient enablement all showed statistically significant improvements after education. Mean adherence with eye drops was maintained above $85 \%$ before and for 3 months after attendance at the educational program. Self-report exaggerated adherence by at least $10 \%$ when compared with the objective Medical Events Monitoring System data, and in fact the kappa agreement was zero.

Conclusion: All questionnaires other than the Short Form-12 were considered to be valuable measures and use of a Medical Events Monitoring System device was considered to be an objective surrogate measure for adherence with eye drops. A multicenter, randomized, controlled equivalence trial of group versus individualized education using adherence as the primary outcome is the next step.

Keywords: group-based education, glaucoma, adherence, patient compliance

\section{Introduction}

Lack of adherence with ocular hypotensive therapy is viewed as a serious contributory factor in the progression of glaucoma. ${ }^{1-3}$ Adherence is defined as the degree to which medication-taking behavior "corresponds with agreed recommendations from a health care provider." It is difficult to be absolutely confident regarding rates of adherence in the literature because different measures of adherence have been employed; however, a systematic review of nine studies reported that $23 \%-60 \%$ of patients studied were nonadherent at 12 months..$^{5}$ Additionally, a proxy that has been used for adherence is persistence. Persistence is defined as time from starting to discontinuing therapy. A recent systematic review of five studies of medical chart reviews reported that, on
Correspondence: Heather Waterman School of Nursing, Midwifery and Social Work, Jean McFarlane Building, Oxford Road, Manchester MI3 9PL, United Kingdom

Tel +44 I6I 3067639

Fax +44 I6I 3067707

Email heather.waterman@manchester. ac.uk 
average, $67 \%$ of patients were persistent after starting their eye drops one year previously. ${ }^{6}$ However, persistence is a coarser and less clinically relevant measure than daily adherence. Research also shows a variety of patterns of nonadherence, in that patients may take small or long breaks from eye drops then resume again, only to be nonadherent again a few days, weeks, or months later. ${ }^{7}$

There are many patient-identified facilitators or barriers to adherence with glaucoma eye drops, which may be amenable to adherence interventions, including forgetfulness, lack of routine, lack of knowledge about glaucoma, and difficulties with drop instillation. ${ }^{8-11}$ The findings of the relevant studies also reveal that individuals often report more than one reason for nonadherence. This suggests that interventions will need to consist of more than education about glaucoma but should include, among other things, practical assistance, such as teaching eye drop instillation technique. While the studies on facilitators or barriers to adherence are helpful, they are not framed in terms of health education needs and cannot be utilized as preparatory work for developing a health education intervention. Health education needs are defined as "any planned activity designed to produce health or illness related learning." 12

Given that in the UK approximately one million patients attend for glaucoma outpatient appointments per annum, ${ }^{2}$ any health education intervention needs to be targeted to those patients in whom the greatest effect may be achieved. One study found that inexperienced patients, ie, those naive to therapy, were significantly more likely to discontinue therapy within 30 days of starting compared with more "experienced" patients. ${ }^{13}$ At least two studies have reported that patients need support and education when first placed on treatment. ${ }^{11,14}$ Altogether, there appears to be a need to deliver interventions to improve adherence early in the patients' trajectory of care.

To date, most interventions have been delivered to single patients. ${ }^{15}$ The interventions delivered to single patients could be described as resource-intensive and may not be viewed as a viable option in the current financial climate. ${ }^{16}$ Arguably, in addition to delivery to single patients, a complementary approach would be to deliver interventions to groups of patients with glaucoma. Prior to undertaking this project, there had been two studies exploring group-based educational interventions in patients with glaucoma, but neither stated whether the intervention was based on patients' health education needs nor did they investigate the effect of the intervention on adherence with eye drops. ${ }^{17,18}$ Therefore, evidence is lacking on what a group-based education intervention should comprise, and whether it is effective in improving adherence.

This paper reports on the second part of an "action research" project which aimed to develop and then pilot a group-based educational intervention to improve adherence with ocular hypotensive therapy. Action research is defined as "a participatory and cyclical process which aims to advance knowledge while executing an improvement in health care practices." ${ }^{19}$ Our previous paper reported the identification of nine patient health education needs regarding adherence with glaucoma treatment. ${ }^{20}$ The current paper details the second stage of that project, and presents data on an exploratory study of the educational resources developed from those health education needs with the intention to use the data to develop a future randomized controlled trial (RCT). ${ }^{21}$

\section{Materials and methods}

Our research objectives originated from the intention to perform a future RCT. The objectives were to:

- assess the rate of recruitment

- measure participants' attendance at the education sessions

- evaluate participant outcomes before and after the education intervention

- assess the most appropriate outcome measures

- measure participants' actual daily percent adherence using a Medical Events Monitoring System (MEMS) device

- understand participants' views on group education.

\section{Study design}

The study was a quasi-experimental before and after study. This design was chosen for the following reasons:

- we did not know whether group-based education would be acceptable to participants

- we did not know whether the content and format of the group-based education were workable

- we did not know whether MEMS would work, because at the time of the start of the study no one had reported using MEMS.

With these unknowns, it was considered unwise at this stage to invest in an RCT, so a before and after design was carried out to inform development of any future RCT. The study received ethics approval from the Manchester ethics committee (09/H10008/4). 


\section{Participants}

Participants were recruited from one large hospital in the North West of England between June 2010 and September 2010. The intention was to be as inclusive as possible. We did not want to restrict the sample to those who were nonadherent because there is no widely accepted level at which nonadherence is deemed unsafe, and adherence behavior is not stable and tends to decline over time although at different rates. ${ }^{5}$

Inclusion criteria were: outpatients attending a glaucoma clinic; age over 18 years; and diagnosis of chronic open angle glaucoma, ocular hypertension, or normal tension glaucoma (it is understood that, in some countries, eye drop use is negotiable in patients with ocular hypertension, but in the UK, if drops are prescribed, the expectation is complete adherence); diagnosed within the last year; hypotensive eye drops prescribed as monotherapy once daily; and angle grades $\geq 2$ using Shaffer's angle grading system. ${ }^{22}$ Exclusion criteria were: secondary glaucoma, angle closure glaucoma, diabetic retinopathy, or any other diseases or ocular surgical procedures capable of affecting intraocular pressure; allergies to ocular medication; unavailability of interpreters; and any condition which prevented participants from learning in groups.

Participants were consecutively recruited into two sequential groups to undertake a group-based education program. A maximum of 16 participants per group were targeted because a systematic review on the effectiveness of groupbased education for patients with type 2 diabetes reported that group sizes of 16-18 were as effective pedagogically as smaller sizes. ${ }^{23}$ Due to the numbers recruited, the program was run twice, so as not to exceed 16 per group, and in order to gain a sufficient sample size to estimate parameters for outcome measures in any future pilot RCT. ${ }^{24}$ By collecting data before and after the intervention, participants acted as their own controls, which typically reduces sources of variability.

\section{Procedures}

During recruitment, patients were invited to attend a groupbased education program to see its effect on eye drop adherence, and if they agreed, full informed consent was taken for the study. Figure 1 shows the flow of participants through the study. Baseline assessments of the outcome questionnaires were taken one month before the group-based educational program and at one and 3 months after delivery of the program. Adherence with eye drops was observed continuously from one month before to 3 months after intervention.

\section{Intervention and follow-up}

An adult learning approach to group-based education seemed the most appropriate for people with glaucoma. ${ }^{25}$ This was because it was in keeping with our health education approach which sought to place emphasis on strengthening and optimizing people's capacities to control their own health. This approach meant that an interactive style of learning would be provided which drew on participants' experiences and allowed them to ask questions (supplementary material) Overall, it was concluded that we could deliver the content in two sessions one week apart, lasting no longer than two and a half hours each. Having two sessions would also enable important messages to be reinforced and be in the realms of participant acceptability of duration. Supplement 1 shows the content of the program alongside the learning approach utilized, both of which were established by patients and health care professionals during the preceding action research study. ${ }^{20} \mathrm{~A}$ glaucoma-trained nurse led the educational program supported by at least one other health professional.

\section{Outcome measures}

Because this was a feasibility study, our objectives were to test measures likely to be utilized within a future RCT on the effects of the group-based education concerning adherence with eye drops. The revised Glaucoma Adherence Questionnaire (GAQ-R), ${ }^{26,27}$ which measures adherence and knowledge of glaucoma, was chosen because we have used this previously in glaucoma adherence studies. The Revised Illness Perception Questionnaire (IPQ-R) ${ }^{28}$ the Beliefs about Medicines Questionnaire (BMQ), ${ }^{29}$ and the Patient Enablement Instrument $(\mathrm{PEI})^{30}$ were chosen because illness representations, beliefs about medicines, and self-control have been found to be related to adherence in other fields as well as in glaucoma. ${ }^{27}$ The general health questionnaire, ie, the Short Form-12, ${ }^{31}$ was administered to identify if there were any changes in the general health of the participants during the study, given that general health may influence adherence. The GAQ-R has 14 items focusing on adherence and knowledge, with a maximum score of 19 ; it has been shown to have good internal consistency (Cronbach's $\alpha 0.90) .{ }^{26,27}$ The IPQ-R was used to assess perceptions and beliefs about glaucoma and has been used widely in other chronic conditions. It has seven subscales (timeline, time cycle, consequences, personal control, treatment control, illness coherence, emotional trauma). The BMQ was employed to measure beliefs about medication, and has two subscales (ie, necessity subscale and concerns subscale). The PEI was used because it measures 


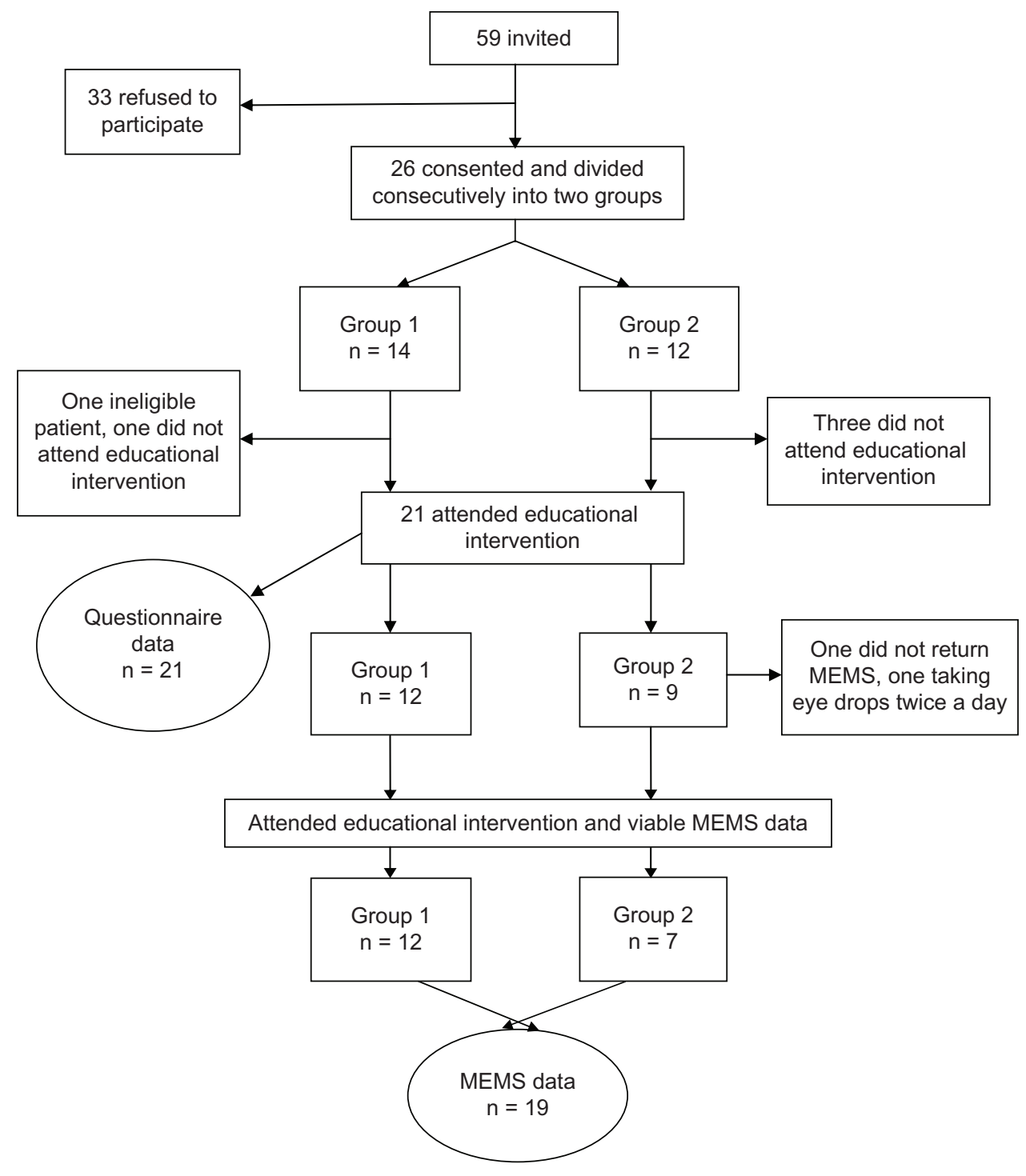

Figure I Flow diagram showing recruitment of participants.

Abbreviation: MEMS, Medical Events Monitoring System.

feelings of empowerment and ability to cope with illnesses and the treatments associated with them. ${ }^{30}$ Scores of up to 12 are achieved on the PEI. Additionally, the Short Form-12 was employed to measure general health. Permission was gained from the authors to make minor alterations to the questionnaires so that items were pertinent to people with glaucoma using eye drops. All questionnaires were sent and returned by post, and were scored as per guidance of the authors who devised the questionnaires. ${ }^{28-31}$

In the absence of any pre-existing gold standard measure, adherence with glaucoma eye drops was measured objectively using a MEMS container. ${ }^{32}$ This is a plastic container with a screw top in which the eye drop bottle is stored until needed for drop instillation. An electronic record is made of the date and time that the top is unscrewed, and this is taken as a surrogate for administering the medication. Participants were told how the MEMS works (including that it recorded when the bottle was opened and that this was being taken as a measure of them taking their eye drops) and were trained how to use it correctly. The MEMS device has previously been tested to measure adherence with eye drops in people with glaucoma. ${ }^{33,34}$ Participant's self-reported adherence was also measured by seven questions requiring a response to a five-item Likert scale in the GAQ-R..$^{26,27}$

\section{Participants' opinion of educational program}

All participants were invited for interviews to evaluate the educational program. In particular, they were asked to focus on those areas of strength and areas for improvement. 
Organizational issues, including barriers to attendance, were included in order to ensure that these could be addressed in any subsequent studies.

\section{Analysis}

The MEMS timestamp data were used to calculate the per participant percentage of adherence in the 4 weeks before the education program, and for one and 3 or more months after the intervention. To allow the participants to settle down with the MEMS, the first 2 weeks of pre-education data were discarded and only data for the 2 weeks preceding the intervention were used. Participants were considered to be adherent if they administered their drops within a window around their previous time of administration ( 2 hours before and 2 hours after). This relative definition of adherence is arguably less harsh and more realistic than an absolute definition of adherence, ${ }^{35}$ because it accommodates when participants might switch from regular morning medication to regular evening medication. By counting the number of adherence events in a period, we also covered for the effect of multiple openings in a short space of time. Individual adherence patterns and collective patterns were scrutinized and plotted. To aid clarity of presentation of the adherence results, doses taken were plotted in the following way because the raw scale plots were uninformative due to outliers dominating the scale. First, the natural $\log (\ln )$ of the interval time between individual doses was calculated in order to reduce the effect of outliers, and then $\ln (24)$ was subtracted to center the plots about an axis of perfect adherence $(\mathrm{y}=1)$. Reference lines were added to show the adherence window. The overall percentage of adherence during a period for each participant was calculated as the fraction of the number of adherent events divided by the number of days. Due to the relatively small sample size, a simple paired $t$-test was used to compare between pre-intervention and post-intervention adherence rates (rather than a generalized estimating equation approach $^{35}$ ). The time of intervention was considered to be immediately after the first session.

Questionnaire scores were analyzed using repeatedmeasures analysis of variance or the equivalent nonparametric Friedman's test as appropriate. Total scores from the questionnaires and dichotomous adherence variables were compared over the three time points, and 95\% confidence intervals (CIs) were calculated. Results were interpreted with caution because the study was not powered to test hypotheses, but were being calculated to inform and provide the basis of a sample size calculation for any future RCT.

To assist with the design of a future RCT, we also compared the rates of adherence between the MEMS data and the GAQ-R to gain insight as to the validity of the Gray et al questionnaire. ${ }^{26,27}$ We descriptively assessed recruitment, attrition, and data collection procedures. ${ }^{24}$ Statistical Package for the Social Sciences version 16 software (SPSS Inc, Chicago, IL, USA) was utilized for all analyses.

\section{Results}

Fifty-nine patients were invited to participate and 26 were recruited. Reasons for nonparticipation included the inability to attend group sessions and not wanting to take part in the research. Two dates were set, and participants were divided consecutively into two groups. Given that this was a feasibility study and in order to maximize the numbers undertaking the educational program, patients were offered another date if they were unable to attend the sessions allocated due to other commitments. One person could not attend the date arranged for session 2 so moved to group 1, hence the unequal numbers per group (Figure 1). Otherwise there was no movement. Group 1 had 14 participants and group 2 had 12 participants. A total of 21 participants attended the educational intervention, and all but one returned the MEMS bottle after taking once-daily medication (Figure 1).

\section{Demographic data}

The average age of the participants who attended the educational intervention was 69 (range 44-89) years and 11 were male. Over half of the participants had retired from full-time work $(n=13)$, three continued to work full-time, two worked part-time, and three were unemployed. According to the medical notes, 14 of the participants had chronic open angle glaucoma, three had ocular hypertension, and four had normal tension glaucoma.

Descriptively, there were no differences between the groups with regard to age, employment status, and diagnosis; however, group 1 consisted mainly of females (75\%) and group 2 consisted mainly of males (88\%). Median adherence pre-intervention was significantly different between the groups (0.93 in group 1 and 0.67 in group 2; $P=0.036$, Mann-Whitney) before intervention, but was not significantly different up to one month (0.87 in group 1 and 0.83 in group 2; Mann-Whitney $P=0.6$ ) or after one month post-intervention ( 0.92 in group 1 and 0.85 in group 2; Mann-Whitney $P=0.2$ ).

\section{Questionnaire data}

The GAQ-R questionnaire demonstrated that participants had a significant increase in their knowledge scores immediately post-intervention $(P<0.0001)$, which was maintained at the 
3-month time point $(P<0.0001)$. Mean scores had changed from 10.1 (score range 4-19) before educational intervention to 14.1 (score range 7-19) immediately post-intervention, and 13.5 (score range 7-19) 3 months post-intervention. See Table 1 for all questionnaire data. All seven subscales of the IPQ-R showed statistically significant differences one month after the educational program $(P=0.002$ to $P<0.0001)$ of at least two points. PEI scores showed significant differences post-intervention compared with before intervention. The mean score before intervention was 4.1 , which increased to 6.6 immediately post-intervention $(P=0.008)$, and rose further to 6.7 at 3 months post-intervention $(P=0.012)$. The BMQ scale only showed significant changes in the subscale of necessity between before and 3 months post-intervention $(P=0.017)$, ie, an increase from 3.7 to 4.1 . Although scores did change in the specific concerns scale, these were not significant $(P=0.3)$. The Short Form-12 showed nonsignificant increases in scores immediately, but these were reversed by the time of the 3-month follow-up. Overall, the Short Form-12 scores showed no significant change.

\section{Adherence data}

MEMS data were analyzed using participants who undertook the education program and returned their MEMS bottles $(\mathrm{n}=19 ; 12$ from group 1 and seven from group 2$)$. One participant from group 2 did not return the MEMS bottle and another (also in group 2) was taking twice-daily eye drop medication so was excluded from the analysis. Four participants did not attend the program (one from group 1 and three from group 2).

The number of days for which eye drop data were collected (after intervention) ranged from 39 days to 99 days (mean 91 days). One participant from group 2 stopped taking the eye drops on day 77 of the study (39 days after intervention). The percentage number of days prior to intervention that the correct dose was taken was $88.7 \%(95 \%$ CI 84.9-92.6), at post-intervention day $1-30$ it was $85.9 \%$ (95\% CI 82.9-88.9), and at post-intervention day 31-99 it was $86.4 \%$ (95\% CI 83.1-89.8). However, this summary may mask individual improvement or worsening of adherence. The time between doses varied considerably, with most clustering within the agreed 4-hour adherence period, but many participants administered doses outside this period. For instance, participant GB5 did not administer a dose for 72 hours part way through the study, whilst participant GB22 had gaps of 220 hours and 260 hours for the last two recorded doses (Figure 2). Most took their eye drops consistently between $7 \mathrm{pm}$ and $2 \mathrm{am}$, while a third took them between 7 am and noon. Figure 3 shows the individual plots of eye drop administration, indicating a variation in time intervals between when the drops were taken each day.

\section{Scheduled doses ( \pm 2 hours)}

The overall participant adherence rate was maintained over time, with the majority remaining approximately $85 \%$ adherent before and after the educational intervention. Adherence was low on the day of intervention (40\%) which is probably an artifact due to participants testing the opening of the MEMS bottle, and afterwards the adherence rate rose sharply. Within the figures are wide daily variations in the mean daily adherence rate (Figure 4). However, towards the end of the study, the variations did appear to have reduced and settled above a mean of $85 \%$. There was no significant difference between pre and post (day 1-30) adherence rates $(t=-0.2$, $d f=18, P=0.8)$, or between pre and later post adherence rates $($ day $31+)(t=-0.1, d f=18, P=0.9)$ when examining the adherence rate on an overall per participant basis; however, the effect of the intervention was different for each individual. Figure 5 plots the individual pre and post overall adherence rates for each participant. Eleven participants improved their adherence from the pre-intervention baseline, whereas seven participants reduced their adherence at both post-intervention time points.

\section{Participant reports of adherence}

Participants were asked about their adherence using the GAQ-R. They were asked how many drops they missed per month $(0,1-3,4-6$, or $7+)$, and the longest period during which they stopped using their drops. The majority $(63 \%)$ said they did not miss any drops, and no participants reported missing more than 4-7 drops at any time, even though some did so according to MEMS data. The adherence rate increased from 63\% (exact 95\% CI 38-83) to 74\% (exact 95\% CI 49-91) one month after the intervention. However, only 58\% (exact 95\% CI 33-80) reported missing no doses 3 months after the educational intervention.

To compare the GAQ data and MEMS data, we calculated the maximum number of drops missed at each time point and the number of person-days to obtain a percentage adherence. This percentage GAQ adherence was conservatively estimated using the highest number of missed doses per range, as $95 \%(=1-[3 \times 5+6 \times 2] /[19 \times 30])$ pre-intervention, $97 \%$ at one month post-intervention, and $95 \%$ at 3 months post-intervention, so participants consistently overestimated their actual adherence as measured by the MEMS. This is further supported when MEMS percent adherence was directly compared with GAQ using 


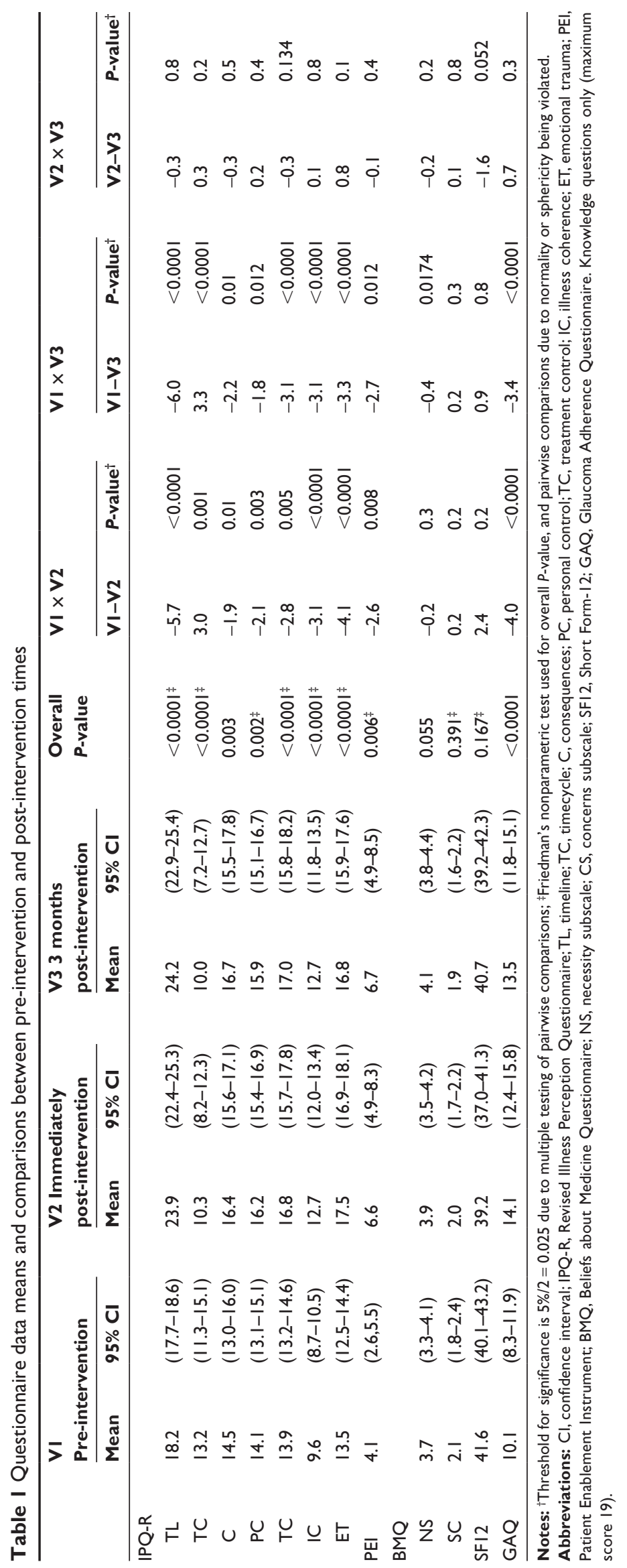




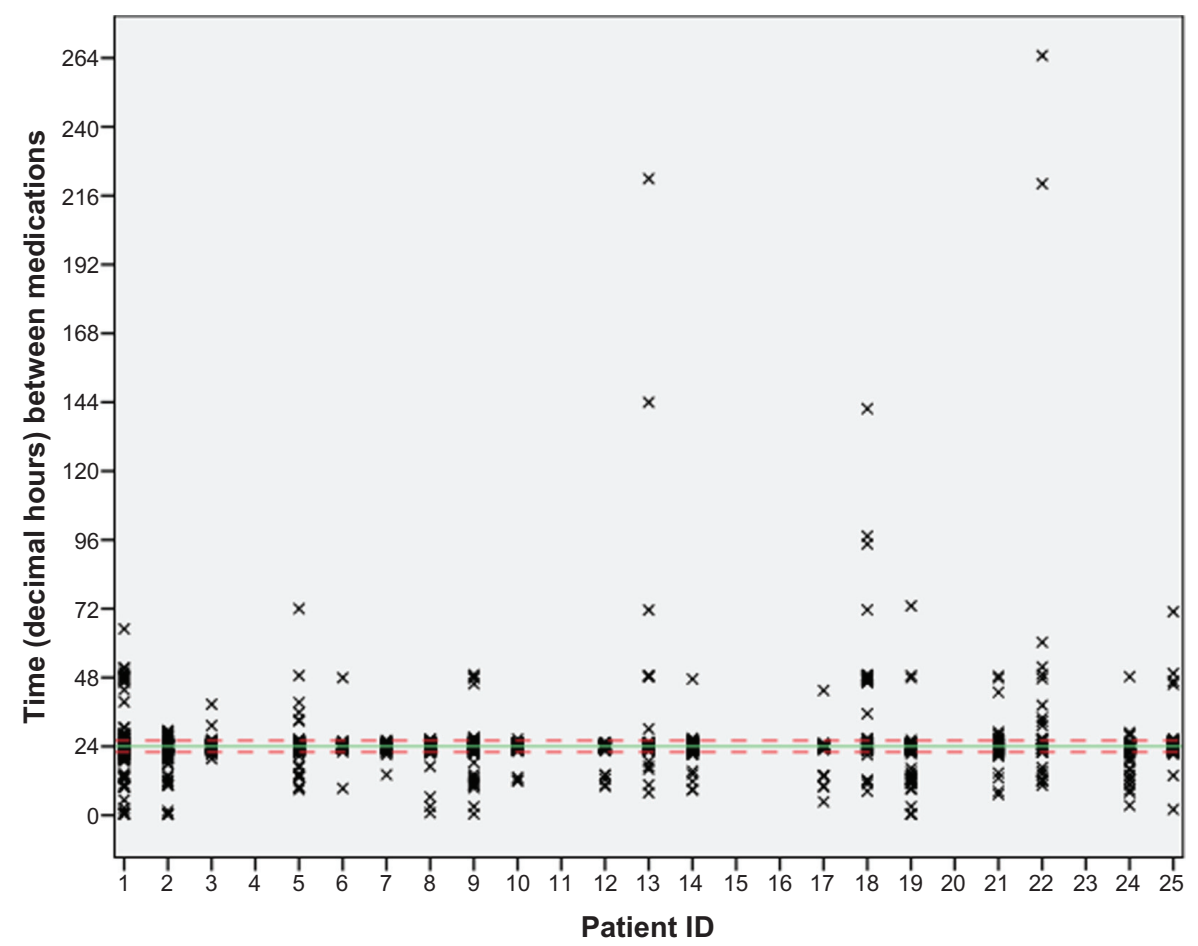

Figure 2 Time between doses for each participant.

Notes: This figure uses all data from 14 days pre-intervention for the 19 participants who completed the educational program. The solid green line is at 24 hours. The dashed red lines are at the adherence boundaries of 22 hours and 26 hours.
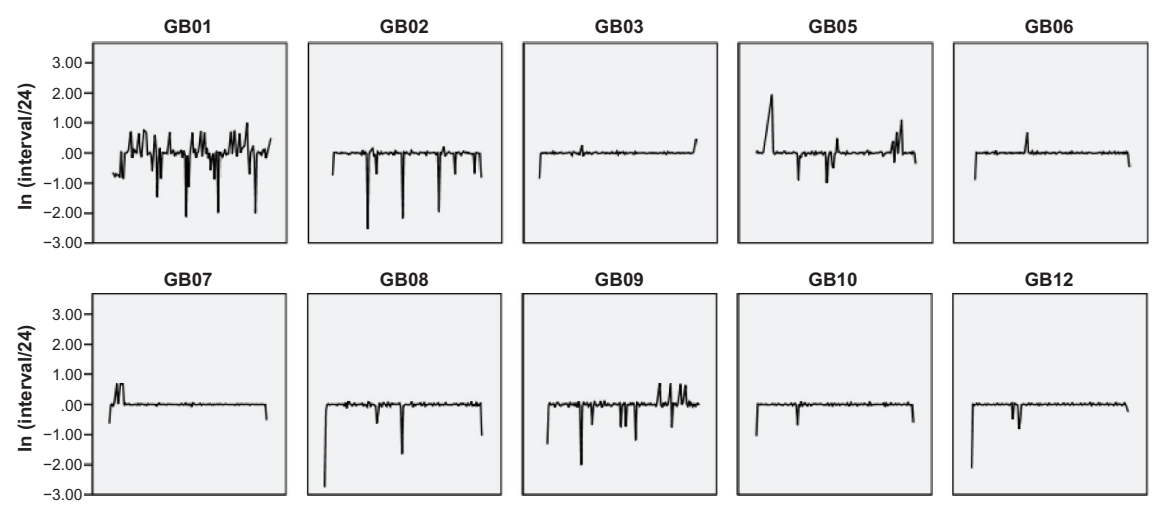

GB09

GB10
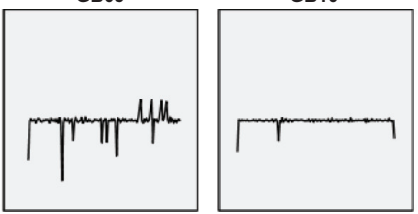

GB12

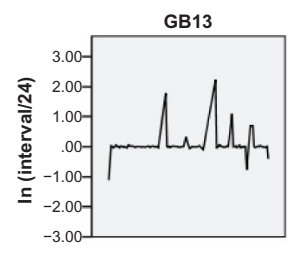

GB14

GB17

GB18
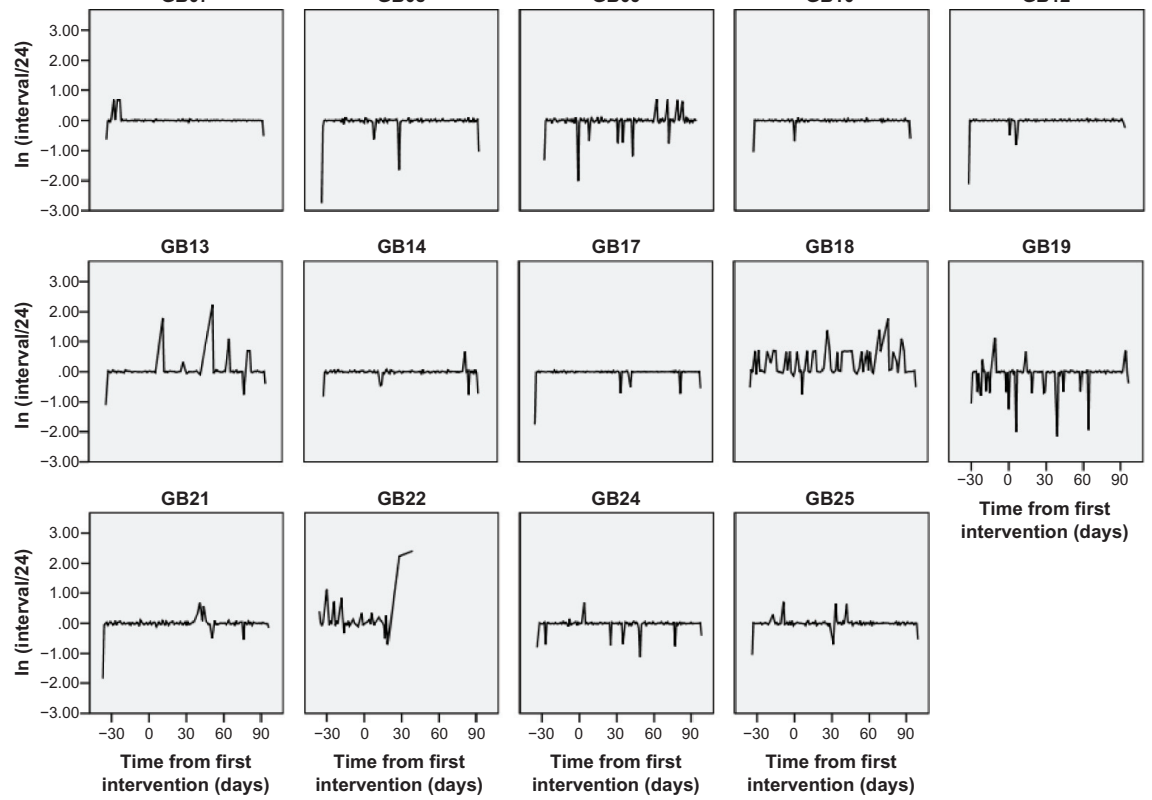

Figure 3 Individual adherence plots (time between drops).

Notes: Time from first intervention. The In (Interval/24) axis has been used to aid presentation of the profiles. Observations on the $y$-axis at zero are perfect adherence (ie, exactly 24 hours since the last medication), while observations away from this line represent loss of adherence.

Abbreviation: GB, group based. 


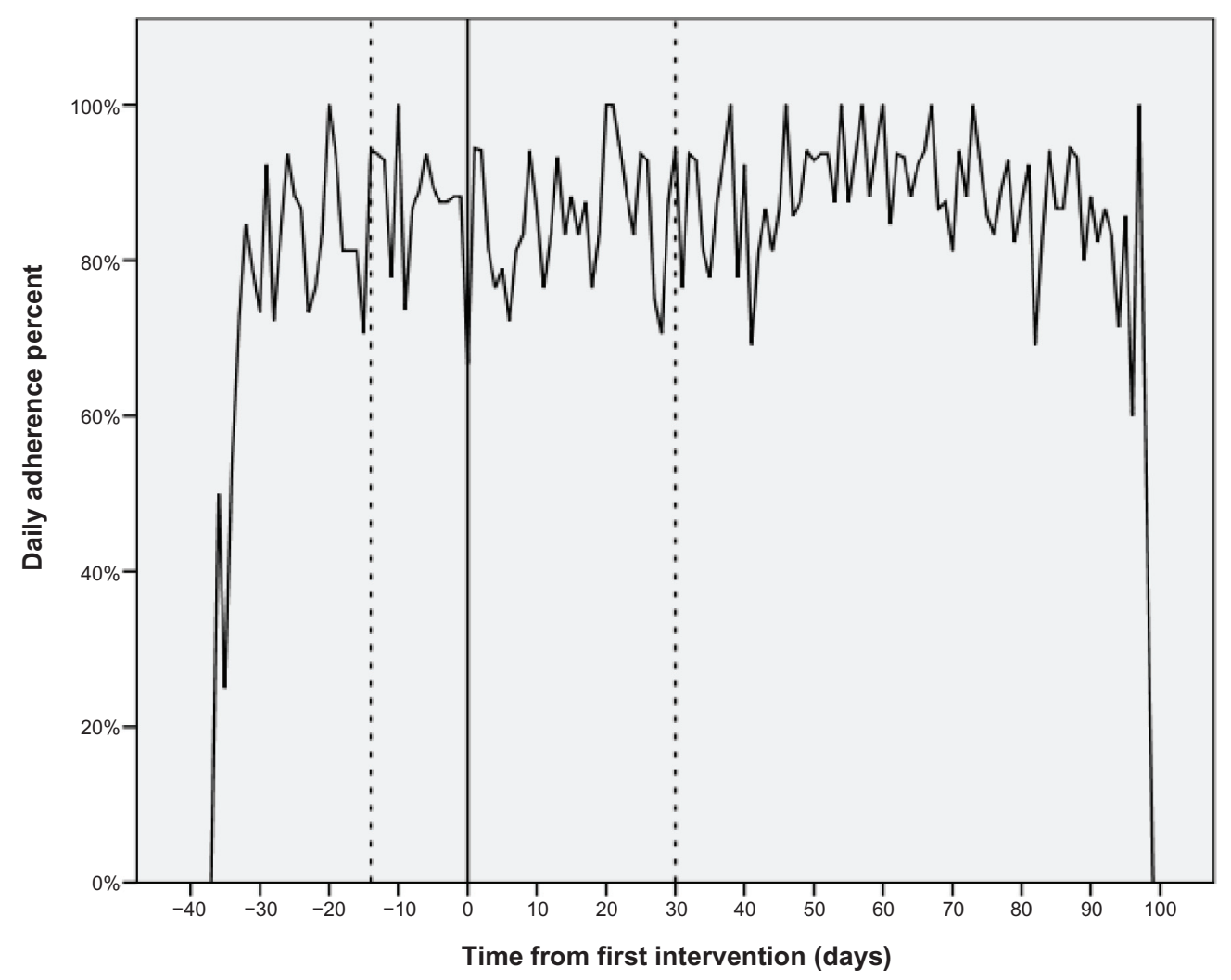

Figure 4 Daily mean adherence rate before and after the intervention.

Notes: Day 0 is the day of the first educational intervention. Daily adherence is the percentage of patients who were adherent on each day.

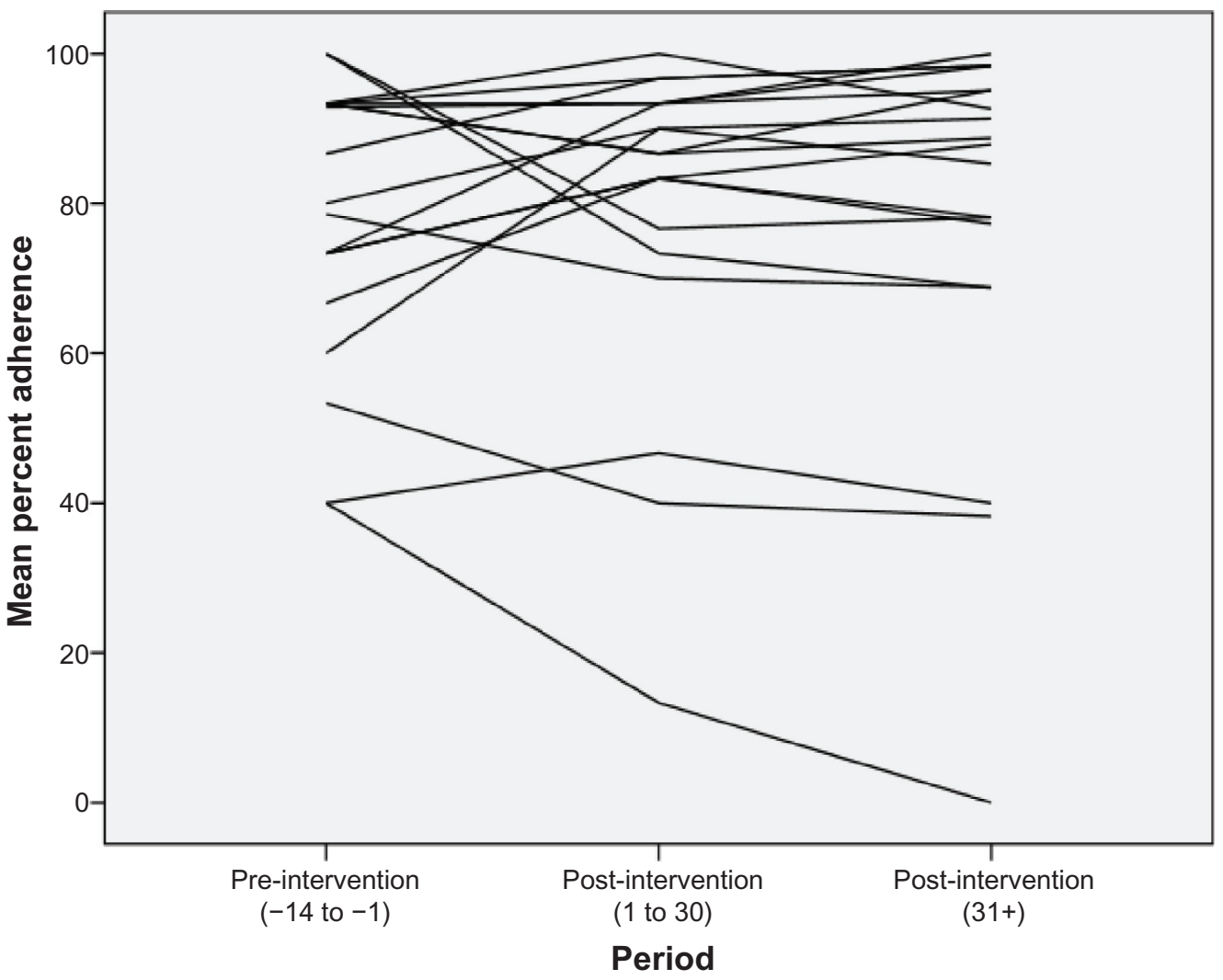

Figure 5 Plot of individual percentage adherence over time (pre-intervention/post-intervention). 
Spearman's correlation. A negative correlation was found between pre-intervention values $(r=-0.37, P=0.1)$ and at one month post-intervention $(r=-0.45, P=0.053)$. At 3 months post-intervention, the correlation was no longer negative but approximately zero $(r=0.043, P=0.9)$. The agreement between MEMS percent adherence and the GAQ was assessed using a weighted kappa statistic, based on a pro rata estimate of monthly MEMS adherence. This statistic was zero for all three time periods, because participants had systematically underestimated the number of doses they missed per month. These figures were used to inform an illustrative calculation for weighted kappa calculation of the sample size required for any future RCT. A sample size of 120 participants would be required to estimate a kappa of 0.1 (with a disagreement rate of 0.8 ) to within an interval of 0.4 with $95 \%$ probability.

\section{Participant perspectives}

Participants reported overwhelming support for the program, identifying how much they had learnt and praising the educator. A few reported that it made them feel more confident to ask questions and instill eye drops. Participants thought that the timing and number of sessions were appropriate.

\section{Discussion}

This study was designed to assess the feasibility for an RCT to assess the effects of a group-based education program on adherence with eye drop therapy. It is the first to look at the relationship between group-based education and illness perception, patient enablement, knowledge of glaucoma, beliefs about medicine, and general health. At the 3-month follow-up, there was a significant improvement in illness perception, patient enablement, knowledge of glaucoma, and in beliefs about medicine. This suggests that the participants felt that they understood their illness more and that they were now capable of taking an active role in their treatment. The BMQ concerns subscale did not show a difference, hence they continued to have concerns over the medication that they were taking but were now more likely to continue taking the eye drops despite this. While these findings need to be treated with caution because of the limitations of a small exploratory before and after design, they support findings from another study which sought to improve adherence through an individualized assessment and plan of action to improve adherence, ${ }^{27}$ and as such confirm that they are valid outcome measures for the future RCT. There was no change in general health as measured by the Short Form-12. This is expected in a chronic condition over such a short period of follow-up and suggests that this measure should not be used in the RCT.

The rate of recruitment was almost identical to that of the forerunner of this study (approximately 45\%), ${ }^{36}$ indicating that there were no unexpected barriers to participation in a group-based education intervention. The attrition rate of $4 / 25(16 \%)$ for attendance at the educational program was reasonable (comparison with previous glaucoma studies on group-based education is difficult because neither study reports attrition ${ }^{17,18}$ ) and only $1 / 25(4 \%)$ did not return the MEMS, the source of data for what is likely to become the primary outcome measure in the RCT making it a useful instrument to measure adherence in future studies. This indicates that recruitment and retention of participants in an RCT is likely to be satisfactory.

During the life of this project, a study of the impact of a single 2-hour education session on persistence of glaucoma treatment was reported..$^{37}$ Blondeau et al found no effect on persistence before and after the education session, which is consistent with our finding that adherence remained level before and after our education program. ${ }^{37}$ Both studies used before and after methods, and were not powered to assess the effectiveness of the group-based intervention. Control groups were not utilized, so it is difficult to draw definitive conclusions. Our focus was on the feasibility of carrying out a trial in the future, and at first sight it could be thought that adherence was not affected and that the trial may not be indicated. However, the data demonstrate that participants who underwent the group-based intervention maintained their initial levels of adherence when normally adherence levels would be expected to decline (irrespective of what measurements are used), ${ }^{6}$ suggesting that an RCT would be meaningful. Decline in adherence should be combated in order to minimize the irreversible progression of glaucoma. A sample size of 120 is suggested, which is manageable given the prevalence of glaucoma.

A gold standard measure of adherence remains elusive, but this experience of using MEMS suggests that it is useful as an objective measure. There remain issues associated with the determination of adherence; we utilized a 4-hour window based on advice from local consultant ophthalmologists, with other researchers in this field using a more lenient measure (ie, an 8-hour window [ \pm 4 hours] of prescribed time ${ }^{7}$ ). Also, a relative definition of adherence (interval between doses) allows for participants to adjust their routine, and is more pragmatic than defining adherence as being assessed against a prescribed (ie, fixed) time of day. However, there is no reason why both windows cannot both be analyzed in future studies in a sensitivity analysis. From a practical point of view 
we found that, to interpret data from the MEMS, it is best to transfer it with the help of a statistician onto a Statistical Package for the Social Sciences database.

Our findings support those of other researchers showing that self-report methods of adherence are unreliable because participants have a tendency to over-report their adherence. ${ }^{38}$ For example, one study found that $76 \% \pm 24.3 \%$ of participants had objectively instilled their eye drops whereas at interview $97.1 \% \pm 5.9 \%$ of the same participants claimed they had been adherent. ${ }^{39}$ Another study found that glaucoma patients tend to have increased social desirability scores compared with their normal counterparts, supporting the propensity for self-report to be unreliable. ${ }^{40}$ Our comparison of the MEMS data and the self-report data suggest that participants tend to overestimate their adherence. A conservative estimate of the mean self-report levels of adherence was 95\%, 97\%, and $95 \%$ at the three time points, but the MEMS recorded respective mean percentages of $89 \%, 86 \%$, and $86 \%$, giving a difference between self-report and an objective measurement of adherence in this study of approximately $10 \%$ at least. This difference is lower than the $20 \%$ identified by Kass et al when studying application of pilocarpine drops using an "unobtrusive eye drop medication monitor" in the 1980s. ${ }^{39}$ This method of summarizing adherence is more granular than using categories and the kappa agreement statistic, which simply showed whether there was disagreement but not in a useful and interpretable way. It is entirely possible that the MEMS data are more accurate due to improvements in technology over the past 30 years.

Because participants were aware that MEMS measured their adherence, it is possible that using it artificially increased adherence in our group. Despite this, adherence levels are within the range reported previously in studies using other forms of adherence measures, so we do not consider it to have affected our results and conclusions.

Although the average adherence was high for glaucoma, the consequences of nonadministration are so adverse (ie, progression to extensive visual impairment), target adherence should be $100 \%$. It might be argued that it would be costeffective to target strategies aimed at improving adherence, such as group-based education, to those who are nonadherent, but it is difficult to know how to identify these patients, because it has been shown that self-report is inaccurate. The clinical advantage of improving adherence in glaucoma is substantial enough to suggest that all patients should be included in future trials.

Data collection procedures proceeded satisfactorily with no difficulties. The process evaluation from the participants' perspective indicated no unintended outcomes or adverse effects. All the data outcome measures appear to produce results that are sensitive to change and, additionally, from previous studies using the IPQ-R, GAQ-R, BMQ, and PEI, ${ }^{27}$ we feel confident that there would be value in using them again in an RCT, except for the self-report measures of adherence.

Prior to the education program, patients in group 2 were less adherent than those in group 1 (67\% versus 93\%; $P=0.036)$ but this variation did not continue after the education programme, and so did not affect our conclusions, given that the comparison was between adherence before and after intervention rather than adherence between groups.

Four participants were recruited who were unable to attend the education intervention due to unforeseen circumstances and the finite time available for this study. A cursory overview of their data shows that their mean adherence prior to the time of intervention was $74.9 \%$ and reduced to $30.2 \%$ at 3 months after the time of intervention, and that their knowledge of glaucoma remained stable between these times. Although an extremely low number of participants were in this group, these data add support to the argument that adherence declines over time without the intervention.

No economic evaluation was undertaken at this stage, because we worked on the premise that group-based education would be more cost-effective than individual education. A future RCT would need to confirm this assumption.

Participant interviews appear to demonstrate that the program met expectations. Participants said that they learnt a great deal and found it enjoyable, and no negative comments were made. They also reported feeling empowered to ask questions of their doctors and were of the opinion that such a program should be part of routine care. Therefore, we conclude, from a participants' perspective, that this intervention warrants further formal testing in an RCT.

Altogether, the findings of this feasibility/pilot study indicate that we should proceed to a full evaluation comprising an assessment of effectiveness and cost-effectiveness and an understanding of change processes, including fidelity to the intervention. ${ }^{21}$ There is a need to compare group-based education with that delivered to single patients, and the findings from this study suggest that an equivalence trial would be most appropriate.

\section{Limitations}

This was a before and after study, with participants acting as their own controls. The results would be strengthened using a separate control group, but this was a pilot study to establish proof of principle. There are potential selection bias issues, eg, 
some participants had been diagnosed with glaucoma for some months and had continued to attend clinics prior to recruitment into the study. It is possible that engagement in clinic attendance and adherence with medication are related, and hence it is possible that we recruited more adherent participants. Additionally, our recruitment rate of 32\% may also suggest self-selection of adherent participants. However, our data show that the adherence profile of the participants was wide-ranging (some adherent and others nonadherent), which is similar to previously published profiles, so we believe that our sample, although small (with low external validity), was representative of a population of medication-takers. The duration of follow-up in our study was 3 months due to funding constraints, so any long-term effects cannot be gauged from these results.

\section{Conclusion}

Education delivered to single patients with glaucoma has been found to be effective in some studies, but no definitive evidence exists to date for the effectiveness of group-based education. A group-based education program was developed and piloted at one hospital in the North West of England. We conclude that the group-based education program may be feasibly delivered in a clinical environment accessible by people with glaucoma, retention is reasonable, and outcomes including the maintenance of adherence appear favorable. Therefore, group-based education is suitable for further evaluation in an equivalence RCT.

\section{Acknowledgments}

We are extremely grateful to the staff and patients who so kindly gave their time to participate in this study. We are also indebted to the Burdett Trust for Nursing, which funded this research. Our thanks also go to the staff at Central Manchester Foundation Trust and Manchester Royal Eye Hospital who hosted and facilitated the study.

\section{Disclosure}

The authors report no conflicts of interest in this work.

\section{References}

1. American Academy of Ophthalmology Glaucoma Panel. Preferred Practice Pattern ${ }^{\circledR}$ Guidelines. Primary Open-Angle Glaucoma. San Francisco, CA: American Academy of Ophthalmology; 2010. Available from: http:// one.aao.org/CE/PracticeGuidelines/PPP.aspx? $\mathrm{p}=1$. Accessed August 21st 2013.

2. National Institute of Health and Clinical Effectiveness. Glaucoma: diagnosis and management of chronic open angle glaucoma and ocular hypertension. London, UK: National Collaborating Centre for Acute Care, Royal College of Surgeons; 2009. Available from: http://www.nice.org. uk/nicemedia/pdf/CG85FullGuideline.pdf. Accessed August 3, 2013.
3. Kotecha A, Fernandes S, Bunce C, Franks WA. Avoidable sight loss from glaucoma: is it unavoidable? Br J Ophthalmol. 2012;96:816-820.

4. World Health Organization. Adherence with long term therapies: evidence for action. Geneva, Switzerland: World Health Organization; 2003. Available from: http://whqlibdoc.who.int/ publications/2003/9241545992.pdf. Accessed August 3, 2013.

5. Lu VH, Goldberg I, Lu CY. Use of glaucoma medications: state of the science and directions for observational research. Am J Ophthalmol. 2010;150:568-574.

6. Reardon G, Kotak Schwartz GF. Objective assessment of compliance and persistence among patients treated for glaucoma and ocular hypertension: a systematic review. Patient Prefer Adherence. 2011;5: 441-463.

7. Ajit R, Fenerty C, Henson D. Patterns and rate of adherence with glaucoma therapy using an electronic dosing aid. Eye (Lond). 2010;24: 1338-1343.

8. Taylor SA, Galbraith SM, Mills RP. Causes of non-compliance with drug regimens in glaucoma patients: a qualitative study. $J$ Ocul Pharmacol Ther. 2002;18:401-409.

9. Tsai JC, McClure CA, Ramos SE. Compliance barriers in glaucoma: a systematic classification. J Glaucoma. 2003;12:393-398.

10. Kholdebarin R, Campbell RJ, Jin YP, Buys YM. Multicenter study of compliance and drop administration in glaucoma. Can J Ophthalmol. 2008;43:454-461.

11. Lacey J, Cate H, Broadway DC. Barriers to adherence with glaucoma medications: a qualitative research study. Eye (Lond). 2008;23: 924-932.

12. Green J, Tones K. Health Promotion: Planning and Strategies. 2nd ed. London, UK: Sage; 2010.

13. Vanelli M, Pedan A, Liu N, Hoar J, Messier D, Kiarsis K. The role of patient inexperience in medication discontinuation: retrospective analysis of medication non-persistence in seven chromic conditions. Clin Ther. 2009;31:2628-2652.

14. Lunnela J, Kaariainen M, Kyngas $H$. The views of compliant glaucoma patients on counseling and social support. Scand J Caring Sci. 2010;24: 490-498.

15. Waterman H, Evans JR, Gray TA, Henson D, Harper R. Interventions for improving adherence with ocular hypotensive therapy. Cochrane Database Syst Rev. 2013;2:CD006132.

16. Haynes RB, Ackloo E, Sahota N, et al. Interventions for enhancing medication adherence. Cochrane Database Syst Rev. 2008;2:CD000011.

17. Blondeau P, Esper P, Mazerolle E. An information session for glaucoma patients. Can J Ophthalmol. 2007;42:816-820.

18. Kim S, Stewart JF, Emond MJ, et al. The effect of a brief education program on glaucoma patients. J Glaucoma. 1997;6:146-151.

19. Waterman H, Tillen D, Dickson R, Koning de K. Action research: a systematic review and assessment for guidance. Health Technol Assess. 2001;5(23).

20. Waterman H, Brunton L, Fenerty C, Mottershead J, Richardson C, Spencer F. Adherence with ocular hypotensive therapy: patient health education needs and views on patient education. Patient Prefer Adherence. 2013;7:55-63.

21. Medical Research Council. Developing and evaluating complex interventions: new guidance. London, UK: Medical Research Council; 2008. Available from:http://www.mrc.ac.uk/ complexinterventionsguidance. Accessed May 24, 2012.

22. Kolker AE, Hetherington J Jr, editors. Becker and Shaffer's Diagnosis and Therapy of the Glaucomas. 5th ed. St Louis, MO: CV Mosby; 1976.

23. Deakin T, McShane CE, Cade JE, Williams RD. Group based training for self-management strategies in people with type II diabetes mellitus. Cochrane Database Syst Rev. 2005;2:CD003417.

24. Lancaster GA, Dodd S, Williamson PR. Design and analysis of pilot studies: recommendations for good practice. J Eval Clin Pract. 2004;10: 307-312.

25. Knowles M. The Adult Learner: A Neglected Species. 4th ed. Houston, TX: Gulf Publishing Company; 1990. 
26. Gray TA, Fenerty C, Harper R, et al. Preliminary survey of educational support for patients prescribed ocular hypotensive therapy. Eye (Lond). 2010;24:1777-1786.

27. Gray TA, Fenerty C, Harper R, et al. Individualized patient care as an adjunct to standard care for promoting adherence with ocular hypotensive therapy: an exploratory randomized controlled trial. Eye (Lond). 2012;26:407-417.

28. Moss-Morris R, Weinman J, Petrie K, Horne R, Cameron L, Buick D. The Revised Illness Perception Questionnaire (IPQ-R). Psychol Health. 2002;17:1-16.

29. Horne R, Weinman J, Hankins M. The Beliefs About Medicines Questionnaire: the development and evaluation of a new method for assessing the cognitive representation of medication. Psychol Health. 1999; $14: 1-24$.

30. Howie JG, Heaney DJ, Maxwell M, Walker JJ. A comparison of a Patient Enablement Instrument (PEI) against two established satisfaction scales as an outcome measure of primary care consultations. Fam Pract. 1998; 15:165-171.

31. Quality Metric. Health Outcomes. SF12v2 Health Survey. Available from: http://www.qualitymetric.com/WhatWeDo/SFHealth Surveys/SF12v2HealthSurvey/tabid/186/Default.aspx. Accessed October 22, 2008.

32. Robin AL, Novack GD, Covert DW, Crockett RS, Marcic TS Adherence in glaucoma: objective measurements of once daily and adjunctive medication use. Am J Ophthalmol. 2007;144:533-540.
33. Sleath B, Blalock S, Covert D, et al. The relationship between glaucoma medication adherence, eye drop technique, and visual field defect severity. Ophthalmology. 2011;118:2398-2402.

34. Sleath B, Blalock S, Covert D, et al. Validation of a short version of the glaucoma medication self-efficacy questionnaire. Br J Ophthalmol. 2012;96:258-262.

35. Düsing R, Handrock R, Klebs S, Tousset E, Vrijens B. Impact of supportive measures on drug adherence in patients with essential hypertension treated with valsartan: the randomized, open-label, parallel group study. VALIDATE. J Hypertens. 2009;27:894-901.

36. Waterman H, Annis G. IGA open summer patient meeting. IGA News. 2011; Winter:15-25.

37. Blondeau P, Carbonneau M, Esper P, Turcotte P-C. A 2-hour information session and patient recall has minimal impact on glaucoma-treatment persistence in a mature practice. J Glaucoma. 2012;21:379-382.

38. Kass MA, Gordon M, Meltzer D. Can ophthalmologists correctly identify patients defaulting from pilocarpine therapy? Am J Ophthalmol. 1986;101:524-530.

39. Kass MA, Meltzer D, Gordon M, Cooper D, Goldberg J. Compliance with topical pilocarpine treatment. Am J Ophthalmol. 1986;101:515-523.

40. Hollo G, Kothy P, Geczy A, Varghaz P. Personality traits, depression, and objectively measured adherence with once-daily prostaglandin analog medication in glaucoma. J Glaucoma. 2009;18:288-292. 


\section{Supplementary Material}

Supplement I Content of a group-based educational program for patients with glaucoma to improve adherence with eye drops

\begin{tabular}{llc}
\hline $\begin{array}{l}\text { Manchester group-based } \\
\text { education program }\end{array}$ & How learning outcome will be met & Duration \\
(Numbers indicate learning outcome) & & (minutes) \\
\hline
\end{tabular}

Session I

For group to chat informally prior

to start of the session

I. To understand the diagnosis/understand difficulties with giving a diagnosis

2. To understand glaucoma

9. To put the condition into perspective - to know how to manage their risk

I. To understand the diagnosis/understand difficulties with giving a diagnosis

2. To understand glaucoma

9. To put the condition into perspective - to know how to manage their risk

3. To understand the implications of eye drops

4. To understand the side effects of eye drops and tablets

5. To feel confident to instill eye drops

8. To understand their own reasons for nonadherence

10. Referring them to other sources of information and support

\section{Session 2}

Tea and coffee prior to start of session

8. To understand their own reasons for nonadherence

6. To be able to ask questions of the doctors/ nurses/optometrists involved in their care

7. To be able to challenge the system
Provide patients with tea, coffee, and biscuits - informal chat

Serve tea/coffee throughout the sessions, so patients

Prior to start

can help themselves

In groups of 3-4, discuss:

- Why are you here today?

- How did you get referred to the eye hospital?

- What happened after you were referred?

- What do you know about your condition?

- What do you want to know?

- What problems (if any) did you encounter in the last month related to either diagnosis or drops?

The professional facilitators will use the examples, identified by patients from the "patient discussion" above to illustrate the following:

- How the eye works

- What is glaucoma? (including eye pressure)

- Difficulties of giving a diagnosis

- Different types of diagnosis

- How might glaucoma affect my eye sight?

- What to expect at an eye clinic appointment (explain tests such as field test, link to eye drops - what have they been prescribed/have drops been changed?)

Linked from previous segment "what to expect at eye clinic appointment"):

- Practice instilling eye drops (practical workshop) - use of aids to help instill eye drops

- Professional facilitator will use examples from previous discussion to :

- explain how eye drops work to control glaucoma

- explain if eye drops interfere with other medicines

- side effects of drops

- Practical issues - eg, should they be kept in fridge/what if I work nights?/do they have to be put in same time each night?

- Start to discuss behavior of using drops/beliefs about drops/medicines in general

Participants are supported to develop their own understanding

of adherence and how they might maintain/improve it. This is

done via group and individual discussions using a "questionnaire"

to facilitate discussion

Professional facilitators hand out information (leaflets)

of session proper

30

Have list of information sources

Book appointment for individual chat with health professional

Facilitator to ask more formal questions of group to assess

if learning outcomes have been met

In group discuss how useful the course has been for them,

good points, points to improve, what would they change?

Group activities - feedback on adherence behavior - how have

their plans to maintain/improve adherence worked?

Practical advice re how to find out information they need in a consultation

Tips “n” tricks + group discussion

- Write down questions you have before you go in to consultation

- Take somebody in to consultation with you so they remember if you don't + opportunity to have individual chat with a health professional for advice 
Supplement I (Continued)

\begin{tabular}{|c|c|c|}
\hline $\begin{array}{l}\text { Manchester group-based } \\
\text { education program } \\
\text { Learning outcome }\end{array}$ & How learning outcome will be met & $\begin{array}{l}\text { Duration } \\
\text { (minutes) }\end{array}$ \\
\hline $\begin{array}{l}\text { 9. To put the condition into } \\
\text { perspective - to know how } \\
\text { to manage their risk }\end{array}$ & $\begin{array}{l}\text { Professional and patient facilitators moderate discussions on: } \\
\text { DVLA regulations for patients with glaucoma } \\
\text { lifestyle and glaucoma }\end{array}$ & 20 \\
\hline Evaluation & $\begin{array}{l}\text { Group discussion } \\
\text { Action plan - what are they going to do differently as a result } \\
\text { of attending the group-based education sessions? } \\
\text { Discuss how useful the course has been for them } \\
\text { Good points, bad points, what would they change? }\end{array}$ & 10 \\
\hline
\end{tabular}

Abbreviation: DVLA, Driver and Vehicle Licensing Authority.

\section{Publish your work in this journal}

Patient Preference and Adherence is an international, peer-reviewed, open access journal focusing on the growing importance of patient preference and adherence throughout the therapeutic continuum. Patient satisfaction, acceptability, quality of life, compliance, persistence and their role in developing new therapeutic modalities and compounds to optimize clinical outcomes for existing disease states are major areas of interest. This journal has been accepted for indexing on PubMed Central. The manuscript management system is completely online and includes a very quick and fair peer-review system. Visit http://www.dovepress.com/ testimonials.php to read real quotes from published authors.

\footnotetext{
Submit your manuscript here: http://www.dovepress.com/patient-preference-and-adherence-journal
} 